\title{
A Case Study of Mesenteric Ischemia by Low Flow CT Imaging
}

\author{
Zacharia Traore ${ }^{*}$, Pierlesky Elion Ossibi², Asmae Zeroual ${ }^{1}$, Seydou Ly ${ }^{1}$, Imane Kamaoui ${ }^{1}$, \\ Youssef Lamrani', Meryem Boubbou', Moustapha Maâroufi', Khalid Mazaz', \\ Siham Tizniti ${ }^{1}$ \\ ${ }^{1}$ Department of Radiology, Hassan II Teaching Hospital, Fes, Morocco \\ ${ }^{2}$ Department of Visceral Surgery, Hassan II Teaching Hospital, Fes, Morocco \\ Email: ztraore84@yahoo.fr
}

Received 17 December 2014; accepted 22 March 2015; published 26 March 2015

Copyright (C) 2015 by authors and Scientific Research Publishing Inc.

This work is licensed under the Creative Commons Attribution International License (CC BY).

http://creativecommons.org/licenses/by/4.0/

(c) (i) Open Access

\begin{abstract}
Acute mesenteric ischemia is a serious disease whose prognosis depends on early diagnosis and therapeutic management. Recent imaging technics play a fundamental role in its diagnosis. There are many forms of mesenteric ischemia including the non-occlusive form which is rare among others. We report the case of a non-occlusive mesenteric ischemia by low flow in a patient with terminal renal disease placed on hemodialysis.
\end{abstract}

\section{Keywords}

Mesenteric Ischemia, Low Flow

\section{Introduction}

Acute mesenteric ischemia is a serious disease that results from a mismatch between, on one hand, perfusion in the territory concerned and, on the other hand, the tissue's metabolic oxygen demand. The prognosis is determined by early diagnosis and therapeutic management. Nonetheless, recent imaging technics play a fundamental role in its diagnosis. This entity forms part of the wider nosology of intestinal ischemia and includes four distinct forms: arterial occlusion by embolus (40\%) or thrombosis (30\%), venous thrombosis (10\%) or non-occlusive form (20\%) [1]. Non-occlusive mesenteric ischemia by low flow remains rare. We report the case of a non-occlusive mesenteric ischemia by low flow in a 50-year-old patient without significant medical history, hospitalized in the nephrology department for end stage renal disease placed on hemodialysis.

${ }^{*}$ Corresponding author.

How to cite this paper: Traore, Z., Ossibi, P.E., Zeroual, A., Ly, S., Kamaoui, I., Lamrani, Y., Boubbou, M., Maâroufi, M., Mazaz, K. and Tizniti, S. (2015) A Case Study of Mesenteric Ischemia by Low Flow CT Imaging. Open Journal of Radiology, 5, 3438. http://dx.doi.org/10.4236/ojrad.2015.51006 


\section{Case}

50-year-old woman with no significant medical history, hospitalized in the nephrology department for end stage renal disease placed on hemodialysis. During hospitalization, the patient complained of abdominal pain, more pronounced in the right iliac fossa associated with fever without any other signs.

On physical examination, patient was conscious had stable vital signs and $38^{\circ} \mathrm{C}$ febrile.

Abdominal examination revealed generalized abdominal guarding more accentuated in the right iliac fossa. The rest of the physical examination was unremarkable.

Laboratory tests came back for a leukocytosis 13,000/. $\mathrm{mm}^{3}$ with CRP 250, with blood urea and creatinine at 1.90 to 110 respectively.

Additional computed abdominal tomography (multi slice CT, spiral acquisition, $5 \mathrm{~mm}$ slice thickness, reconstructed in to $2.5 \mathrm{~mm}$ ) with intravenous iodinated contrast during the arterial and portal phases revealed a large hypodense lesion measuring 3.5 by $2.9 \mathrm{~cm}$ located in segment VII of the liver with a peripheral base and extending towards the hilum relating to hepatic ischemia and as well as spontaneously hyper dense adrenal glands concordant with hypovolemic shock (Figure 1 and Figure 2). This was associated with pneumatosis intestinal is in the right colon wall and discrete densification of the surrounding fat coupled with mild reactive bowel wall thickening in the small intestine measuring $6 \mathrm{~mm}$ in large diameter (Figure 3).

The patient underwent surgery with per operative discovery of extensive ischemic necrosis of small bowel extending up to the right colon without signs of perforation. Right colectomy was performed. The postoperative period was uneventful with favorable clinical and biological parameters.

\section{Discussion}

The term "ischemic colitis” was first used by Marston in 1966 [2]. It refers to an anoxia of the large bowel wall of circulatory origin, which involves several mechanisms usually without vascular occlusion [3]-[5].

Two types have been identified according to their prognosis, the most common form:

- Non gangrenous ischemic colitis: moderate severity, reflects damage to the mucosa and submucosa which usually regresses with medical treatment,

- Gangrenous ischemic colitis: the rarest form entails a trans-parietal affection associated with peritoneal signs, requiring surgical treatment and with poorer prognosis [3] [4].

Clinical signs are highly dependent on the mechanism and cause of ischemia: the more complete and quicker the setting in of ischemia, the more severe the signs. In general, clinical presentation involves abdominal pain and general tenderness without guarding in the early stages. At an advanced stage of ischemia, it usually involves guarding and other signs of peritoneal irritation, shock and or bowel obstruction. Melena or hematochezia were observed in $15 \%$ of cases demonstrating mucosal damage [6].

In case of non-occlusive ischemia, diagnosis is based on the combination of the following 3 signs: acute ab-

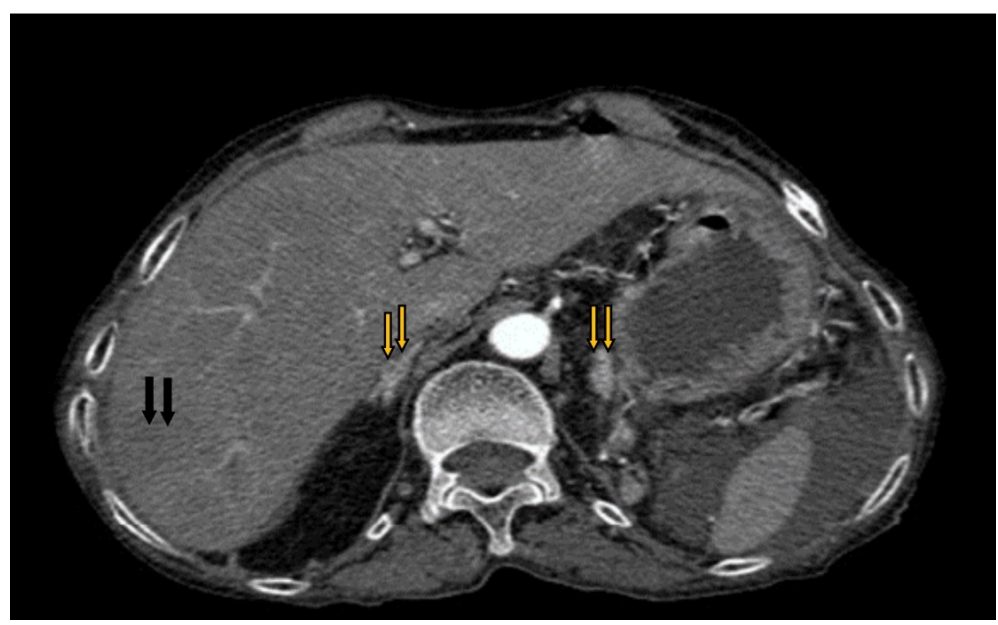

Figure 1. Axial section in arterial phase showing hypodense lesion in segment VII of the liver $(\checkmark)$ with spontaneously hyper dense adrenal glands $(\downarrow \downarrow)$. 


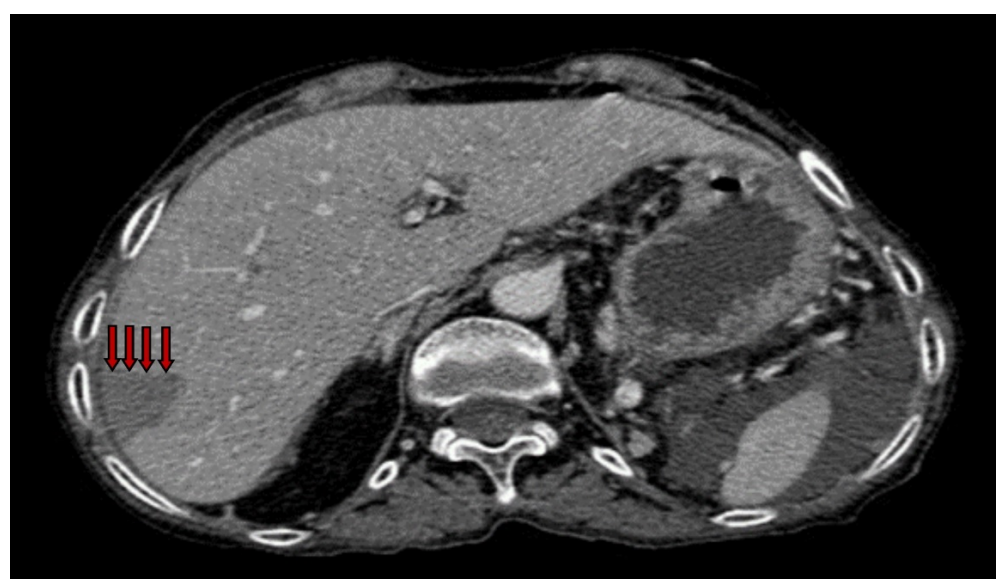

Figure 2. Axial section at the portal phase showing a clearly evident hypo dense lesion in segment VII of the liver.

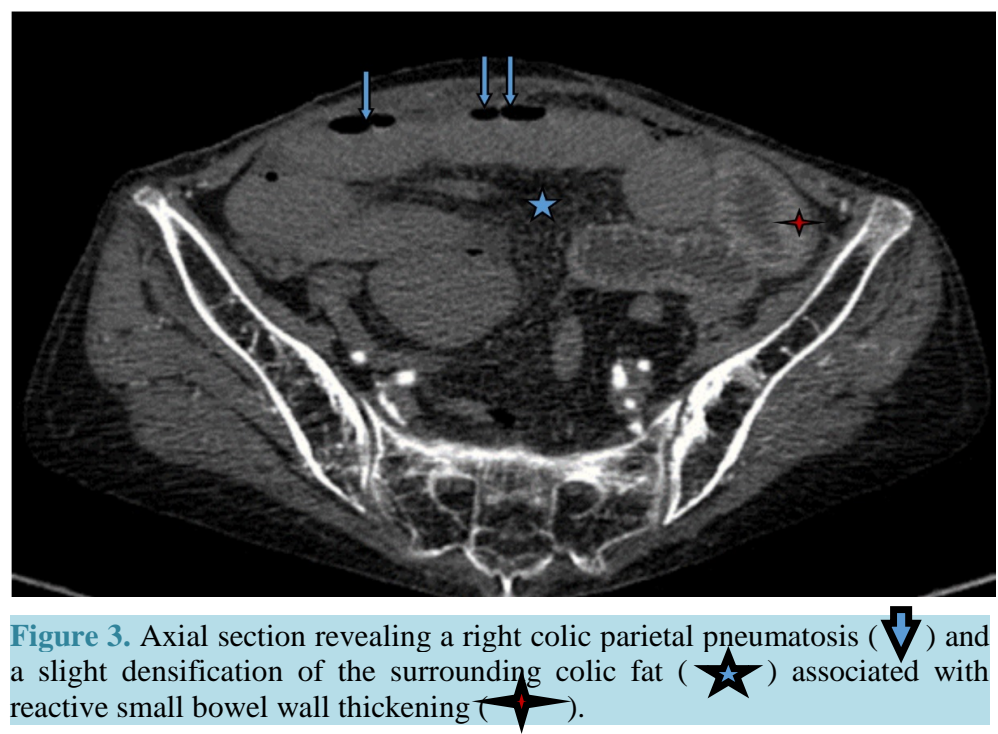

dominal pain; acute gastro intestinal disorder (inflammatorysyndrome), intestinal hypoperfusion syndrome.

Multi slice tomography with arterial, venous and portal phase acquisitions, plays a major role because it allows the positive diagnosis, determines etiology and severity of acute mesenteric ischemia, thus defining its management.

Its diagnostic contribution in the study of the aorta, the coelio mesenteric trunks and their immediate branches of division is excellent. It may detect complete or incomplete occlusions of the superior mesenteric artery and vein.

Topographically, lesions observed are in relation to the distribution of arterial vascularization, particularly at the junction of the upper and lower mesenteric territories (left colic angle) and anastomotic area between the territories of the inferior mesenteric artery and iliac artery (rectosigmoid junction).

It usually affects the left colon and sigmoid colon (50\% of cases) [5] [7]. Right colic cases have also been reported in low flow ischemia [8]. This discovery reveals two interesting points.

The first concerns the peculiar topography and severity of ischemic colitis in hemodialysis patients. The preferential involvement of the right colon in hemodialysis patients has been reported [9] [10]. In a series of 60 patients with ischemic colitis, right colic locations was noted in $82 \%$ of hemodialysis patients while it was only found in $26 \%$ of other patients [10]. Also the right colic location is associated with more severe forms as evidenced by $62 \%$ of patients requiring surgical treatment had a right colonic involvement as against only $19 \%$ of medically treated patients [10]. This confirms that ischemic colitis is an important cause of morbidity and mor- 
tality in hemodialysis patients [11]. The pathogenesis of the right colon involvement in these patients is unclear [12]. The entire right colon is vascularized by 3 colic branches of the superior mesenteric artery (upper, middle and lower colic arteries) which anastomose with each other. Two factors could be taken into account to explain the right predominance of ischemia. First vascularization of the cecum is provided by the terminal branches of the lower colic artery, the anterior and posterior cecal arteries [13]. On the other hand, the right colicvasarecta are longer than the left colic vasa recta [12]. The repeated occurrence of episodes of hypotensioninhemodialysis patients could result in vasoconstriction of rights colicvasarecta with difficulty in subsequent reperfusion once the hypotension is resolved [10]. The second point concerns the remarkable CT signs of the disease. The dominant role of computed tomography compared with colonoscopy in ischemic colitis is clearly established [5] [6] [14], especially inhemodialysis patients [10]. However, CT signs are not very specific in mild ischemia where only a thickening of the colon wall is most often observed, probably associated with a lack of blood supply [5].

Management of mesenteric ischemia entails emergency surgery coupled with medical treatment: volemic resuscitation and preoperative antibiotherapy in preparation to explorative surgery. Surgery remains the sole curative treatment which consists of resection of the necrotized portion. In addition, it entails verification of the superior mesenteric arteryin order to perform either balloon catheter embolectomy or even endarterectomy is sometimes a useful revascularization technic. However, revascularization by bridging is rarely indicated. Mortality rate of mesenteric ischemia still remains shockingly high ranging from $60 \%$ to $90 \%$ : a rate that has not changed considerably in the past decade.

The peculiarity presented in this case highlights two interesting points:

- The particular topography and severity of ischemic colitis in chronic hemodialysis patients

- The CT semiotics of the disease.

\section{Conclusions}

Entero-mesenteric ischemia by low flow is a rare affection. Its diagnosis should be considered based on clinical suspicion with current imaging technics playing a vital role in confirming its diagnosis.

The emergency treatment is usually surgical with good prognosis.

\section{References}

[1] Brandt, L.J. and Boley, S.J. (2000) AGA Technical Review on Intestinal Ischemia. American Gastrointestinal Association. Gastroenterology, 118, 954-968. http://dx.doi.org/10.1016/S0016-5085(00)70183-1

[2] Marston, A., Pheils, M.T., Thomas, M.L. and Morson, B.C. (1966) Ischaemic Colitis. Gut, 7, 1-25. http://dx.doi.org/10.1136/gut.7.1.1

[3] Toursarkissian, B. and Thompson, R.W. (1997) Ischemic Colitis. Surgical Clinics of North America, 77, 461-470. http://dx.doi.org/10.1016/S0039-6109(05)70561-2

[4] Robert, J.H., Mentha, G. and Rohner, A. (1993) Ischaemic Colitis: Two Distinct Pattern of Severity. Gut, 34, 4-6. http://dx.doi.org/10.1136/gut.34.1.4

[5] Balthazar, E.J., Yen, B.C. and Gordon, R.B. (1999) Ischemic Colitis: CT Evaluation of 54 Cases. Radiology, 211, 381388. http://dx.doi.org/10.1136/gut.34.1.4

[6] Chang, R.W., Chang, J.B. and Longo, W.E. (2006) Update in Management of Mesenteric Ischemia. World Journal of Gastroenterology, 12, 3243-3247.

[7] Horton, K.M., Corl, F.M. and Fishman, E.K. (2000) CT Evaluation of the Colon: Inflammatory Disease. Radiographics, 20, 399-418. http://dx.doi.org/10.1148/radiographics.20.2.g00mc15399

[8] Flynn, T.C., Rowlands, B.J., Gilliland, M., Ward, R.E. and Fischer, R.P. (1983) Hypotension-Induced Post-Traumatic Necrosis of the Right Colon. The American Journal of Surgery, 146, 715-718. http://dx.doi.org/10.1016/0002-9610(83)90325-2

[9] Jablonski, M., Putzki, H. and Heymann, H. (1987) Necrosis of the Ascending Colon in Hemodialysis Patients. Diseases of the Colon \& Rectum, 30, 623-625. http://dx.doi.org/10.1007/BF02554811

[10] Flobert, C., Cellier, C., Berger, A., et al. (2000) Right Colonic Involvement Is Associated with Severe Forms of Ischemic Colitis and Occurs Frequently in Patients with Chronic Renal Failure Requiring Hemodialysis. The American Journal of Gastroenterology, 95, 195-198. http://dx.doi.org/10.1111/j.1572-0241.2000.01644.x

[11] Diamond, S.M., Emmett, M. and Henrich, W.L. (1986) Bowel Infarction as a Cause of Death in Dialysis Patients. Journal of the American Medical Association, 256, 2545-2547. http://dx.doi.org/10.1001/jama.1986.03380180107028 
[12] Landreneau, R.J. and Fry, W.J. (1990) The Right Colon as a Target Organ of Nonocclusive Mesenteric Ischemia. Archives of Surgery, 125, 591-594. http://dx.doi.org/10.1001/archsurg.1990.01410170037007

[13] Simon, A.M., Birnbaum, B.A. and Jacobs, J.E. (2000) Isolated Infarction of the Cecum: CT Findings in Two Patients. Radiology, 214, 513-516. http://dx.doi.org/10.1148/radiology.214.2.r00fe15513

[14] Wiesner, W., Khurana, B., Ji, H. and Ros, P.R. (2003) CT of Acute Bowel Ischemia. Radiology, 226, 635-650. http://dx.doi.org/10.1148/radiol.2263011540 